\title{
Enterovirus 71 induces autophagy by regulating has-miR-30a expression to promote viral replication
}

Yuxuan Fu ${ }^{\mathrm{a}}$, Deyan Chen ${ }^{\mathrm{a}}$, Wentao Xua ${ }^{\mathrm{a}}$, Chunhong Feng ${ }^{\mathrm{a}}$, Xiaohui Wang ${ }^{\mathrm{a}}$,Li Zhang ${ }^{\mathrm{a}}$, Xiaowen Lv ${ }^{\mathrm{d}}$, Nan Zheng ${ }^{\mathrm{a}, \mathrm{b}, \mathrm{c}}$,

\author{
Yu Jin $^{\mathrm{d}}$, Zhiwei Wu $\mathrm{u}^{\mathrm{a}, \mathrm{b}, \mathrm{c}}$
}

${ }^{\mathrm{a} C e n t e r}$ for Public Health Research, Medical School, Nanjing University, Nanjing;

${ }^{\mathrm{b}}$ State Key Lab of Analytical Chemistry for Life Science, Nanjing University, Nanjing;

${ }^{\mathrm{c}}$ Medical School and Jiangsu Key Laboratory of Molecular Medicine, Nanjing University, Nanjing, P.R. China;

dNanjing Children's Hospital, Nanjing Medical University, Nanjing, PR China 


\begin{abstract}
Enterovirus 71 (EV71), the etiological agent of hand-foot-and-mouth disease, has increasingly become a public health challenge around the world. Previous studies reported that EV71 infection can induce autophagic machinery to enhance viral replication in vitro and in vivo, but did not address the underlying mechanisms. Increasing evidence suggests that autophagy, in a virus-specific manner, may function to degrade viruses or facilitate viral replication. In this study, we reported that EV71 infection of human epidermoid carcinoma (Hep2) and African green monkey kidney cells (Vero) induced autophagy, which is beneficial for viral replication. Our investigation of the mechanisms revealed that EV71 infection resulted in the reduction of cellular miR-30a, which led to the inhibition of Beclin-1, a key autophagy-promoting gene that plays important roles at the early phase of autophagosome formation. We provided further evidence that by modulating cellular miR-30a level through either overexpression or inhibition, one can inhibit or promote EV71 replication, respectively, through regulating autophagic activity.
\end{abstract}

\title{
Keywords:
}

Enterovirus 71; autophagy; miR-30a; Beclin-1; viral replication 


\section{Introduction}

Enterovirus 71 (EV71), first identified and isolated from the feces of an infant patient with aseptic meningitis in 1969 in California, has caused a number of life-threatening outbreaks around the world, particularly in the Asia-Pacific region (Solomon et al., 2010). EV71 is an etiological agent for hand-foot-and-mouth disease (HFMD) in young children. EV71 infection is self limiting in most of the cases but in some patients the infection will lead to neurological manifestations and death(Wong et al., 2010; McMinn, 2002). The pathogenesis of EV71 is poorly understood and no vaccine or specific antiviral therapy is available.

Autophagy plays important physiological and pathological roles in maintaining cellular homeostasis through eliminating mutated/misfolded aggregated proteins and damaged organelles in double-membrane structures called autophagosomes. The process of autophagosome formation involves two major procedures: nucleation and elongation of the isolation membrane ( $\mathrm{He}$ and Klionsky, 2009). In the beginning, the formation of the initial membrane nucleation phase requires a kinase complex including Beclin-1, a BH3-only protein, which is used as a marker for monitoring autophagy (Gannage et al., 2010). During the elongation of the autophagosome membrane, the cytosolic microtubule-associated protein 1 light chain 3 beta (LC3B)-I is converted to LC3B-II through modification into phosphatidylethanolamine-conjugated form. The LC3B-II is the only protein marker that is reliably associated with completed autophagosomes (Yoshimori, 2007).

The autophagy induced by viral infections was reported in the 1960s and the potential significance of its roles in viral infection is increasingly being appreciated (Dales et al., 1965). Viruses, such as HIV, herpes simplex virus type I, dengue virus, hepatitis C virus, influenza A virus and coxsackievirus B3, have been shown to induce autophagy (Campbell and Spector, 2013; Lussignol et al., 2013; Lee et al., 2008; Ait-Goughoulte et al., 2008; Zhou et al., 2009; Wong et al., 2008). Evidence suggests that autophagy, in a virus-specific manner, may function to either restrict or facilitate viral replication (Jheng et al., 2014; Richards and Jackson, 2013; Munz, 2011). Virus-induced autophagy may provide a scaffold for virus replication or for evading immune 
surveillance. Previous report showed that EV71 infection could induce autophagic machinery to enhance viral replication in vitro and in vivo (Huang et al., 2009), and Liu et al demonstrated that EV71-infected suckling mice showed an autophagic flux in the brain tissues (Lee et al., 2014). However, the underlying mechanisms that trigger autophagy by EV71 are not known. Unraveling the mechanisms may provide new clues to understand the pathogenesis of EV71 infection, allowing discovery of potential antiviral agents and strategies.

In an effort to elucidate regulatory mechanisms of EV71-induced autophagy, we sought to explore the roles of microRNAs (miRNAs) in the regulation of cellular autophagy processes, which are important modulators of gene expression at the post-transcriptional level by binding to 3'UTR region of mRNAs (Skalsky and Cullen, 2010; Cullen, 2009). Currently, the expression of host miRNAs during EV71 infection is the focus of much interest, for instance, miR-296-5p, miR-146a and miR-27a, which were shown to be associated with EV71 infection or replication (Zheng et al., 2013; Ho et al., 2014; Zhang et al., 2014b). More importantly, substantial evidence confirms functional roles for miRNAs in pathways of autophagy (Zhai et al., 2013; Chen et al., 2014). Herein, we hypothesize that host miRNAs might be involved in EV71-induced autophagy through modulating the relevant genes.

In conclusion, we identified a miRNA, miR-30a, that inhibited EV71 replication by modulating EV71-induced autophagy. Equally important is that the down-modulation of cellular miR-30a facilitated EV71 replication, suggesting that this microRNA play important roles in the EV71-induced autophagosome formation. It would be interesting to further investigate the mechanism of the EV71-mediated miR-30a expression and to explore the potential therapeutic value of miR-30a for EV71 infection.

\section{Materials and methods}

\subsection{Cell cultures and drug treatments}

Hep2 (Human epidermoid carcinoma) and Vero cells (African green monkey kidney cells) were obtained from American Type Culture Collection (ATCC, USA) and were cultured in Dulbecco's 
modified Eagle's medium (DMEM) containing $10 \%$ fetal bovine serum (FBS) in a $37^{\circ} \mathrm{C}$ humidified atmosphere of $5 \% \mathrm{CO}_{2}$. The autophagy inhibitor wortmannin was purchased from Sigma and used at the final concentrations of $100 \mathrm{nM}$ and $300 \mathrm{nM}$ in Vero and Hep2 cells, respectively. Cells were infected, or mock-infected, and drugs were added to the medium after the first hour of infection.

\subsection{Western blot}

The lysed cells were subjected to SDS-polyacrylamide gel electrophoresis, transferred onto PVDF membranes(Millipore, MA, USA) and incubated with appropriate primary and secondary antibodies. Membranes were scanned by using Li-COR Odyssey Infrared Imager (Li-COR Bioscience). All the results were quantified by densitometric analysis using an Odyssey software. Primary antibodies against Beclin-1 (Santa Cruz, CA, USA), LC3B (Cell Signaling Technology, USA), p62 (Cell Signaling Technology, USA), $\beta$-actin (Bioworld Technology, Minnesota, USA), VP1 (Millipore, Billerica, MA) were used.

\subsection{RNA extraction, reverse transcription, and qRT-PCR analysis}

Total RNA, including miRNA, was extracted from cell lysate with TRIzol reagent (Life Technologies) according to the manufacturer's instructions. Reverse transcription and real-time polymerase chain reaction was performed as previously described(Zheng et al., 2013). The sequences of mRNA primer pairs were as follow:

EV71VP1: Forward: 5'-GCTCTATAGGAGATAGTGTGAGTAGGG-3'

Reverse: 5'-ATGACTGCTCACCTGCGTGTT-3'

Beclin-1: $\quad$ Forward: 5'-CAAGATCCTGGACCGTGTCA-3'

Reverse: 5'-TGGCACTTTCTGTGGACATCA-3'

\subsection{Virus infection and titration}

EV71 Fuyang0805 strain was a kind gift from Dr. Bin Wu, Jiangsu Provincial Centers of Disease Control and propagated on Vero cells. Briefly, confluent Vero cells at a multiplicity of infection (m.o.i.) of 1 in DMEM containing 2\% fetal bovine serum and the EV71 virus stocks were collected from the supernatants of infected cells at day 4 postinfection (p.i.). The titers of the viral 
stocks were determined by the $50 \%$ tissue culture infectious dose $\left(\mathrm{TCID}_{50}\right)$ assay on Vero cells and stored at $-80^{\circ} \mathrm{C}$. As of the $\mathrm{TCID}_{50}$ assay, serially diluted viruses from $10^{-2}$ to $10^{-10}$ in $2 \%$ FBS containing DMEM were inoculated to Vero cells in 96-well plates. The cytopathic effect (CPE) in infected Vero cells was observed under the microscope after 4 days. Virus titer was determined by Reed-Münch endpoint calculation method (Heaton and Randall, 2011).

\subsection{Plasmid transfection}

Vero or Hep2 cells were seeded onto 6-well plates and incubated until the confluence reached about $80 \%$. The cells were then transfected with a plasmid expressing GFP-LC3 using Lipofectamine 3000 according to the manufacturer's protocols. 12 hours later, the cells were infected with EV71 virus at $0.03 \mathrm{TCID}_{50}$ for indicated times and the percentage of the cells showing the LC3 punctate aggregation was counted under a fluorescence microscope. Cells containing $\geq 4$ punctate GFP-LC3 localization were defined as autophagy-positive cells. The number of autophagy-positive cells relative to GFP-expressing cells was considered to indicate the differences.

\subsection{Transmission electron microscopy}

Vero cells infected with EV71 $\left(0.03 \mathrm{TCID}_{50}\right)$ were fixed with $2 \%$ glutaraldehyde in $0.1 \mathrm{M}$ cacodylate buffer, pH 7.4 plus $5 \mathrm{mM} \mathrm{CaCl}$, rinsed in buffer, postfixed in $1 \% \mathrm{OsO}_{4}$ in $0.1 \mathrm{M}$ cacodylate buffer for $60 \mathrm{~min}$ in $4^{\circ} \mathrm{C}$, washed three times with distilled water, stained with aqueous $2 \%$ uranyl acetate for $30 \mathrm{~min}$, then the cell were photographed under a Hitachi 7000 transmission electron microscope (Hitachi, Tokyo, Japan) operated at $75 \mathrm{kV}$. The average number of autophagic vesicle (AV) per cell was evaluated. A minimum of 50 cells were observed. Cell counting was done by three independent experiments and data are presented as mean \pm SEM.

\section{7 miRNA transfection and confocal microscopy}

Vero or Hep2 cells were transfected with GFP-LC3 in a 24-well plate using the Lipofectamine3000 according to the manufacturer's instructions and incubated for 12 hours. The final concentration of pre-miR-30a or antago-miR-30a and their negative control (RiboBio, Guangzhou, China) was 100nM per well. After 24h, the cells were infected with EV71 virus at the 
$0.03 \mathrm{TCID}_{50}$ for various time durations and then examined under the fluorescent microscope. The fluorescence of GFP-LC3 was analyzed and the images were acquired.

\subsection{Luciferase assays}

The 3'-UTR of Beclin-1 was amplified by PCR using human genomic DNA and cloned into the Xbal site downstream of firefly luciferase genes in the pGL3 vector (primer forward: 5'-GCTCTCAAGTTCATGCTGACGAAT-3'; Reverse: 5'-CAGTTTTCAGACTGCAGCAAATCT-3'). A pGL3 construct containing 3'-UTR of Beclin-1 with mutant seed sequences of has-miR-30a was also synthesized. For luciferase assay, Vero and Hep2 cells were seeded in 96-well plates and transfected with the luciferase reporter plasmid containing 3'UTR of Beclin-1, the control plasmids (pRL-TK Vector; Promega), and either hsa-miR-30a mimic or a negative control (miR-n.c). The cells were collected after $48 \mathrm{~h}$ using the dual-Luciferase reporter assay system (Promega, WI, USA). All experiments were performed five times and the results were expressed as firefly luciferase activity normalized to renilla luciferase activity.

\subsection{Immunofluorescence staining}

Immunofluorscence staining was performed according to standard procedures. Briefly, at various times post-infection, cells were fixed with $3.7 \%$ paraformaldehyde and permeabilized in $0.1 \%$ Triton X-100 and sequentially incubated with primary and fluorophore-tagged secondary antibodies. DAPI dyes (Beyotime Institute of technology, China) was used for cell nucleic acid stains. The samples were examined under a fluorescence microscopy and images were analyzed using an Olympus FluoView FV10i confocal microscope (Tokyo, Japan). Data points presented in the text are from six different fields.

\subsection{Plaque assay}

Virus titers in the supernatant fraction were measured as previously described (Bird et al., 2014)

\subsection{Statistical Analysis}

Data from at least three independent experiments were presented as mean \pm SEM using SPSS 19.0 
statistical software. The difference among treatment groups were analyzed by Student's t-test or One-way ANOVA followed by Student-Newman-Keuls (S-N-K) test. P values from a two-tailed test were $<0.05$ were considered statistically significant.

\section{Results:}

\subsection{EV71 induced autophagy activity in Hep2 and Vero cells.}

Previous reports showed that EV71 infection could induce autophagy activity and then promoted its own replication in human rhabdomyosarcoma RD and neuronal SK-N-SH cells (Huang et al., 2009; Lee et al., 2014); however, the mechanism of EV71 triggering autophagy is unknown. Moreover, it is possible that autophagy may be dependent on cell types. To determine if autophagy was induced by EV71 virus in Hep2 and Vero cells, the two cell lines were transfected with green-fluorescent LC3 plasmid (GFP-LC3), and 12h after transfection the cells were infected with or without EV71 and were treated with wortmannin (a Ptdlns 3-kinase inhibitor that blocked autophagy at autophagosome formation stage) at 6,12 and 24hpi. In contrast to the diffused expression pattern of GFP-LC3 in mock-infected cells, the number of GFP-LC3 punctate-positive cells was accumulated in the EV71-infected cells starting 6hpi and reached the peak at 24hpi (Fig. 1A). Wortmannin treatment resulted in a significant reduction of the percentage of GFP-LC3 puncta-positive cells (18\% in Hep2 cells, $20 \%$ in Vero cells) and, typically fewer punctate aggregates were observed in wortmannin-treated than non-treated cells, suggesting the inhibition of the activity of EV71-induced autophagy. To further confirm the observations, infected cells were examined by transmission electron microscopy (TEM) and quantitative analysis was performed for the autophagosome-like vesicles in EV71-infected Vero cells. We observed that the number of double or single membrane vesicles increased more than 10 fold in the cytoplasm of the infected Vero cells but rarely in the mock-infected cells (Fig. 1B). These results provided evidence that autophagy was induced in both Vero and Hep2 cells by EV71 infection. At the same time, we also detected colocalization of LC3 and VP1 (EV71 structural protein) in EV71-infected Hep2 cells under confocal microscopy, further supporting the evidence that EV71 infection induces autolysosome formation (Fig. 1C).

3.2 Protein expressions relevant to autophagic activity were altered upon EV71 infection and 


\section{autophagy facilitated viral replication}

To further confirm that EV71 infection indeed induced autophagy, autophagic specific protein markers, Beclin-1 and LC3, were investigated to determine autophagy activation by Western blot analysis in Hep2 and Vero cells. The conversion of LC3-I to LC3-II is generally considered to be a reliable indicator of autophagy and the ratio of LC3-II/LC3-I is widely used to measure the autophagic flux (He and Klionsky, 2009; Yoshimori, 2007). As shown in Fig. 2A and B, the Beclin-1 and LC3-II protein expression gradually increased as the infection proceeded from 6 to 24 hpi, indicating that EV71-induced autophagy activity in both Vero and Hep2 cells (Fig. 2A and B; lanes 3, 5 and 7). To illustrate the influence of autophagy on EV71 replication, we used wortmannin during EV71 infection. The presence of wortmannin resulted in lower expression of Beclin-1, LC3-II and VP1 in the EV71-infected cells than the infected cells without wortmannin treatment (Fig. 2A and B, lanes 4, 6 and 8). Beclin-1 expression was minimally affected by wortmannin in the mock treated cells. In addition, we measured extracellular virus production in the culture supernatant of infected Vero and Hep2 cells when autophagy was inhibited by wortmannin at different time points postinfection. The extracellular EV71 titers were markedly reduced by the presence of wortmannin at 6,12 , and $24 \mathrm{~h}$ after infection (Fig. 2C and D), suggesting that the inhibition of autophagy inhibited the viral replication.

\subsection{EV71 infection down-regulated expression of hsa-miR-30a.}

Recently, Cui et al. reported that more than sixty miRNAs were involved in the host response to EV71 infection Hep2 cells by a deep sequencing approach (Cui et al., 2010). Among these miRNAs, the down-regulated miRNA-30a, was shown to negatively regulate Beclin-1 expression and result in decreased autophagic activity in some diseases(Zhu et al., 2009; Zou et al., 2012; Yin et al., 2013; Pan et al., 2013;Wang et al., 2014). To confirm the expression of miR-30a in EV71-infected cells, we measured the expression patterns of this miRNA in EV71-infected Vero and Hep2 cells by qRT-PCR. Another miRNA, hsa-miR-18a, was used as a control. The relative expression level of miR-30a in infected Vero cells was reduced compared to the mock-infected cells at 6,12 and 24 hpi. (Fig. 3A). Similarly, the expression level of miR-30a in infected Hep2 was also reduced(Fig. 3B), while the level of miR-18a was not affected significantly by EV71 infection(Fig.3A and B). To further investigate the relationship between miR-30a and autophagy, 
we determined the expression of miR-30a when autophagy was inhibited by wortmannin. We found that inhibition of autophagy by wortmannin resulted in an overall up-regulation of miR-30a expression in both cell lines, and smaller reduction of miR-30a in infected cells relative to the un-treated mock control, while the expression of miR-18a did not change significantly when autophagy was inhibited by wortmannin. Taken together, these results indicated that the cellular miR-30a expression was significantly inhibited during the course of EV71-induced autophgy, which was consistent with the previous report by Cui et al that the expression level of miR-30a was down-regulated in EV71-infected Hep2 cells.

\section{4 miR-30a targeted and inhibited Beclin-1}

Beclin-1, a key autophagy-promoting gene that participates in the early stage of autophagosome formation, is negatively regulated by miRNA-30a, leading to the decreasing autophagic activity in tumor cells (Zhu et al., 2009; Zou et al., 2012) and cardiomyocytes (Yin et al., 2013; Pan et al., 2013). Therefore, we investigated if miRNA-30a regulating autophagic activity during EV71 infection is mediated by Beclin-1. Using a miRNA target prediction database (TargetScan 6.0, Diana microT 3.0 and miRanda), we found that miR-30a has the seed region that matches the 3'UTR of Beclin-1 (nucleotides 98-105, Fig. 4A). To verify whether Beclin-1 gene is a target for miR-30a, we cloned luciferase reporter vectors containing either wild-type or deletion mutant Beclin-1 3'UTR sequences downstream of the luciferase coding sequence and the respective luciferase activities were determined when the cells were co-transfected with either pre-miRNA-30a (a chemically synthetic oligonucleotide that designed to mimic endogenous mature miRNA molecules when transfected into cells) or pre-miR-n.c that served as a negative control. As shown in Fig. 4B and C, the miR-30a dramatically reduced the relative luciferase activity of the reporter construct containing wild-type Beclin-1 3'UTR in both Vero and Hep2 cells compared to the negative control (pre-miR-n.c). In contrast, the luciferase activity in the mutated Beclin-1 3'UTR reporter vector did not show changes, suggesting that the miR-30a target site is 98-105 in Beclin-1 3'UTR. Next, we examined the effect of the pre-miR-30a and antago-miR-30a on autophagic response. Antago-miR-30a contains the complete sequence complementary to endogenous miR-30a and can inhibit miRNA activity through the RNA interference pathway. The results showed that the relative expression levels of miR-30a in Hep2 
and Vero cells transfected with pre-miR-30a were 25- and 40-fold in Hep2 and Vero cells, respectively. (Fig. 4D) while the level of miR-30a in cells transfected with its antagomir was down-regulated by about 60\% (Hep2 cells) and 70\% (Vero cells), respectively. Fig. 4 showed that when cells were transfected with pre-miR-30a, significant reductions of Beclin-1expression were observed in both proteins(Fig. 4E and F) and mRNA levels (Fig. 4G). Conversely, the cells transfected with antago-miR-30a exhibited both increased Beclin-1 protein and mRNA levels. Western blot also showed that the up-regulated miR-30a resulted in a decrease in the ratio of LC3-II/LC3-I and down-regulated miR-30a led to a increase of the ratio(Fig. 4E and F), suggesting that miR-30a modulated autophagic activity. To further substantiate the observation, we assayed the level of the autophagic substrate p62 protein following pre-miR-30a and its antagomir treatment and showed that p62 decreased progressively when miR-30a was upregulated by the exogenous pre-miR-30a, and increased gradually when a blockade of endogenous miR-30a by antago-miR-30a (Fig. 4E and F). These data provided evidence that the miR-30a binding to the 3'-UTR of Beclin-1 contributed to the modulation of Beclin-1expression and suppressed the EV71-induced autophagic activity.

\subsection{Overexpression of hsa-miR-30a inhibited EV71 replication through affecting autophagic activity}

Previous studies shown that stimulation of autophagy increased the extracellular yield of poliovirus , but did not affect the accumulation of intracellular virus RNA. (Bird et al., 2014). To investigate whether hsa-miR-30a inhibited EV71 release or replication, Hep2 cells were transfected with pre-miR-30a, followed by infection with EV71. The extracellular and intracellular infectious virus production harvested from the pre-miR-30a-transfected and infected Hep2 cells, measured at different times postinfection, were all significantly lowered than the cells transfected with pre-miRNA-n.c (Fig. 5A and B). Consistent with the viral titer measurement, the immunofluorescence analysis showed that in the infected cells with miR-30a overexpression the viral capsid protein VP1 decreased at all time points postinfection when compared to the VP1 expression in the cells transfected with pre-miRNA-n.c (Fig. 5C). These results suggested that miR-30a could markedly repress EV71 replication. 
Western blot analysis of VP1 protein expression further confirmed the suppressive effect of miR-30a on EV71 replication. As shown in Fig. 5D, the EV71 VP1 protein levels were reduced by $78 \%$ and $80 \%$ in the pre-miR-30a-tranfected cells at 12 and $24 \mathrm{hpi}$, respectively. At the same time, we also investigated the effect of overexpressed miR-30a on regulating autophagy induction by EV71. Our finding showed that the overexpression of miR-30a blocked EV71-induced autophagosome formation, downregulated the expression of Beclin-1 protein and inhibited the conversion from LC3-I to LC3-II. As expected, we found that the infected cells showed dramatically decreased punctate staining of GFP-LC3 following pre-miR-30a treatment compared to the cells transfected with pre-miRNA-n.c or non-transfected control (Fig. 5E). Together, we concluded that miR-30a could suppress EV71 replication by blocking EV71-induced autophagy.

\subsection{Inhibition of hsa-miR-30a promotes EV71 replication.}

To further illustrate the effect of miR-30a on EV71 replication, Hep2 cells were transfected with the antago-miR-30a to inhibit the has-miRNA-30a expression. Differences in viral titers and expression levels of VP1 were determined (Fig. 6A and B). These results showed that blocking of miR-30a enhanced the EV71 replication and increased EV71 VP1 protein expression, compared to the control cells that were transfected with antago-miR-n.c or mock-treated. Consistent results were also observed by immunofluorescence assay that the numbers of infected cells in downregulated miR-30a group increased compared to the negative control at all times points postinfection (Fig. 6C). We thus conclude that blocking of cellular miR-30a could promote EV71 replication.

\section{Discussion}

Many positive-strand RNA viruses can induce rearrangement of host intracellular membrane compartments during replication. In recent years, research has suggested that these phenomena may be linked to the autophagic response by the host cells. Autophagy is a basic catabolic mechanism that degrades unnecessary or dysfunctional cellular components through lysosomes (Klionsky et al., 2012; Baehrecke, 2005) and its activation protects cells against cytosolic pathogens. However, viruses have also evolved means to exploit the host's autophagy for their 
own benefit (Jheng et al., 2014; Richards and Jackson, 2013; Taylor and Jackson, 2009).

Accumulating evidence has shown that picornavirus infection could induce the formation of membrane-enveloped structures or autophagosomes (Skalsky and Cullen, 2010; Schlegel et al., 1996). Previous studies showed that EV71 infection could induce an autophagy response to enhance its own replication in human rhabdomyosarcoma RD and neuronal SK-N-SH cells (Huang et al., 2009). Further investigation of the signaling pathway revealed that reduced expressions of phosphorylated mTOR and phosphorylated p70S6K were involved in EV71-induced autophagy in a cell-specific manner while extracellular signal-regulated kinase (Erk), PI3K/Akt and Beclin-1 were not (Huang et al., 2009; Lee et al., 2014). In our study, we found that EV71 infection resulted in the up-regulation of Beclin-1 and conversion of LC3-I to LC3-II. The discrepancy of our observations on the roles of Beclin-1 may be due to the use of different cells. Beclin-1, a key initiator of autophagy in mammalian cells, is part of the type III phosphatidylinositol 3-kinase (PI3K) complex and plays an important role in autophagosome formation. Inhibition of PI3K by specific inhibitor, such as wortmannin, has been shown to lead to inhibition of autophagy (Pattingre et al., 2008; Mizushima et al., 2010). Our data showed that wortmannin inhibited EV71-induced autophagy as evidenced by the changes in expression of Beclin-1, LC3-II/LC3-I ratio and GFP-LC3 staining pattern. Beclin-1 contains a conserved Bcl-2 or $\mathrm{Bcl}-\mathrm{X}_{\mathrm{L}}$ binding $\mathrm{BH} 3$ domain, and its apoptotic signaling activity is thought to be mediated by its association with the Bcl-2 family members (Gannage et al., 2010; Munz, 2011). Evidence showed that EV71-infected cells underwent the process of cell death, especially the programmed cell death (PCD), which including apoptosis, autophagy and necrosis (Zhang et al., 2014a). Similar results were also observed in our experiment, where the infected cells underwent apoptosis or death after 72h EV71 infection (data not shown). Xi et al. recently showed that inhibition of autophagosome formation reduced the apoptosis induced by EV71 infection (Poojary et al., 2013), and moreover, researches have demonstrated that autophagy constitutes a stress adaptation that avoids cell death, or constitutes an alternative cell-death pathway (Eisenberg-Lerner et al., 2009; Zalckvar et al., 2010; Gonzalez-Polo et al., 2005; Baehrecke, 2005), suggesting that autophagy and apoptosis might be triggered by common upstream pathway signals.

Numerous researches have concluded that autophagosome structure may serve as a scaffold for viral replication in cytosol, particularly positive-strand RNA viruses like EV71 (Ait-Goughoulte et 
al., 2008; Richards and Jackson, 2013; Huang et al., 2009; Taylor and Jackson, 2009; Xu et al., 2014). For instance, Huang et al. showed that in EV71 infection LC3 and EV71 VP1 proteins surrounded the double-membrane autophagosomes (Huang et al., 2009). In this study, we demonstrated that the LC3-II could be co-localized with EV71 capsid protein VP1, supporting the hypothesis that autophagosomal membrane may serve as a platform for EV71 replication complexes and enhance virus replication.

As an important effector that regulates host's signaling pathways, including those of autophagy, miRNAs are involved in the host response to viral infection (Skalsky and Cullen, 2010; Cullen, 2009; Zhai et al., 2013; Chen et al., 2014;). Increasing evidence has shown that a number of cellular miRNAs were involved in the regulation of EV71 infection and pathogenesis, including miR-146a, miR-296-5p, and miR-1246 (Zheng et al., 2013; Ho et al., 2014; Xu et al., 2014). It was reported that the expression level of miR-30a was down-regulated in EV71-infected Hep2 cells using a deep sequencing technology (Cui et al., 2010). In this study, we confirmed this finding by real-time PCR in EV71-infected Hep2 and Vero cells, and showed that the intracellular expression of miRNA-30a was indeed gradually inhibited during the course of infection. Previous studies have shown that miRNA-30a inhibits autophagy in ischemic stroke, cardiomyopathy and some carcinomas by downregulating Beclin-1 expression (Zhu et al., 2009; Zou et al., 2012; Yin et al., 2013; Pan et al., 2013; Wang et al., 2014; Yu et al., 2012). Recently, a report by Kumar et al. demonstrated that miR-30a was involved in modulating autophagosome formation induced by Hepatitis B virus $\mathrm{X}$ protein $(\mathrm{HBx})$, and that this effect was mediated via reduced Beclin-1 expression (Kumar et al., 2015). Our data showed that miR-30a was downregulated by EV71-induced autophagy mediated by Beclin-1 and further demonstrated that Beclin-1 contains a potential binding site for miRNA-30a. By using the miR-30a mimic and antagomir to either inhibit or up-regulate miR-30a, we further illustrated that miR-30a was able to modulate autophagy through modulating Beclin-1 expression. Wortmannin inhibition of autophagosomes and its impact on miR-30a levels provided further evidence on the roles of miR-30a in autophagic activity.

To explore the role of miRNA-30a in EV71 replication, progeny viral titers and autophagy levels were analyzed by transfecting cells with miR-30a mimic or antagomir. Recently, Bird et al 
demonstrated that stimulation of autophagy could increases the extracellular yield of poliovirus, but not affected intracellular virus RNA, and showed that stimulating autophagy increased frequency of nonlytic viral spread(Bird et al., 2014). Others, some evidence support that it is likely that poliovirus intercepts the autophagy pathway downstream of beclin-1, using components such as ATG12 and LC3 that are required to build double-membraned vesicles(Jackson et al., 2005; Suhy et al., 2000).Our data showed that miR-30a could suppress EV71 intracellular replication and extracellular release through blocking EV71-induced autophagy mediated by miR-30a-regulated Beclin-1 expression. The analysis on extracellular/intracellular progeny viral titer and GFP-LC3 plasmid transfection further confirmed that miR-30a inhibited autophagic activity and inhibited EV71 infection. Taken together, our studies confirmed that EV71 infection induced autophagy through inhibition of endogenous miRNA-30a. Although the mechanisms of EV71 down-regulating miRNA-30a expression are not clear, our observations suggest the existence of a complex physiological interplay between cellular miRNAs and EV71 infection in the host cell.

EV71 infection in humans can lead to neurological manifestations, which is considered to be the major factor in most fatal cases (Solomon et al., 2010; Wong et al., 2010; McMinn, 2002; Chen, 2004). Previous studies in vivo and in vitro, using the autophagosome marker GFP-LC3, indicated that autophagosomes are scarce in healthy neurons (Mizushima et al., 2010; Nixon et al., 2000), and accumulating evidence suggests that autophagy levels have a direct impact on developmental and neurodegenerative processes (Nikoletopoulou et al., 2015; Yamamoto and Yue, 2014; Giordano et al., 2014). Recent finding suggested that EV71 infection of suckling mice induces an amphisome formation accompanied with the autophagic flux in the brain tissues (Lee et al., 2014). Therefore, autophagy may play important roles in the neuropathogenesis of EV71 infection and miR-30a may provide a potential therapeutic target.

\section{Acknowledgements}

This study was supported by the Major Research and Development Project from National Health and Family Planning Commission (Grants No. 2012ZX10001-007-009-001 and 
2013ZX10001005-003), and by Jiangsu National Science Foundation (Grant No: BK20130591).

\section{References}

Ait-Goughoulte, M., Kanda, T., Meyer, K., Ryerse, J.S., Ray, R.B., Ray, R., 2008. Hepatitis C virus genotype 1a growth and induction of autophagy. Journal of virology 82, 2241-2249.

Baehrecke, E.H., 2005. Autophagy: dual roles in life and death? Nature reviews. Molecular cell biology 6, 505-510.

Bird, S.W., Maynard, N.D., Covert, M.W., Kirkegaard, K., 2014. Nonlytic viral spread enhanced by autophagy components. Proceedings of the National Academy of Sciences of the United States of America 111, 13081-13086.

Campbell, G.R., Spector, S.A., 2013. Inhibition of human immunodeficiency virus type-1 through autophagy. Current opinion in microbiology 16, 349-354.

Chen, Y., Fu, L.L., Wen, X., Liu, B., Huang, J., Wang, J.H., Wei, Y.Q., 2014. Oncogenic and tumor suppressive roles of microRNAs in apoptosis and autophagy. Apoptosis : an international journal on programmed cell death 19, 1177-1189.

Chen, Y.C., Yu, C.K., Wang, Y.F., Liu, C.C., Su, I.J., Lei, H.Y., 2004. A murine oral enterovirus 71 infection model with central nervous system involvement. The Journal of general virology 85, 69-77.

Cherry, S., 2009. VSV infection is sensed by Drosophila, attenuates nutrient signaling, and thereby activates antiviral autophagy. Autophagy 5, 1062-1063.

Cui, L., Guo, X., Qi, Y., Qi, X., Ge, Y., Shi, Z., Wu, T., Shan, J., Shan, Y., Zhu, Z., Wang, H., 2010. Identification of microRNAs involved in the host response to enterovirus 71 infection by a deep sequencing approach. Journal of biomedicine \& biotechnology 2010, 425939.

Cullen, B.R., 2009. Viral and cellular messenger RNA targets of viral microRNAs. Nature 457, $421-425$.

Dales, S., Eggers, H.J., Tamm, I., Palade, G.E., 1965. ELECTRON MICROSCOPIC STUDY OF THE FORMATION OF POLIOVIRUS. Virology 26, 379-389.

Eisenberg-Lerner, A., Bialik, S., Simon, H.U., Kimchi, A., 2009. Life and death partners: apoptosis, autophagy and the cross-talk between them. Cell death and differentiation 16, 966-975. 
Gannage, M., Ramer, P.C., Munz, C., 2010. Targeting Beclin 1 for viral subversion of macroautophagy. Autophagy 6, 166-167.

Giordano, S., Darley-Usmar, V., Zhang, J., 2014. Autophagy as an essential cellular antioxidant pathway in neurodegenerative disease. Redox biology 2, 82-90.

Gonzalez-Polo, R.A., Boya, P., Pauleau, A.L., Jalil, A., Larochette, N., Souquere, S., Eskelinen, E.L., Pierron, G., Saftig, P., Kroemer, G., 2005. The apoptosis/autophagy paradox: autophagic vacuolization before apoptotic death. Journal of cell science 118, 3091-3102.

Heaton, N.S., Randall, G., 2011. Dengue virus and autophagy. Viruses 3, 1332-1341.

He, C., Klionsky, D.J., 2009. Regulation mechanisms and signaling pathways of autophagy. Annual review of genetics 43, 67-93.

Ho, B.C., Yu, I.S., Lu, L.F., Rudensky, A., Chen, H.Y., Tsai, C.W., Chang, Y.L., Wu, C.T., Chang, L.Y., Shih, S.R., Lin, S.W., Lee, C.N., Yang, P.C., Yu, S.L., 2014. Inhibition of miR-146a prevents enterovirus-induced death by restoring the production of type I interferon. Nature communications 5,3344 .

Huang, S.C., Chang, C.L., Wang, P.S., Tsai, Y., Liu, H.S., 2009. Enterovirus 71-induced autophagy detected in vitro and in vivo promotes viral replication. Journal of medical virology 81, $1241-1252$.

Jackson, W.T., Giddings, T.H., Jr., Taylor, M.P., Mulinyawe, S., Rabinovitch, M., Kopito, R.R., Kirkegaard, K., 2005. Subversion of cellular autophagosomal machinery by RNA viruses. PLoS biology 3, e156.

Jheng, J.R., Ho, J.Y., Horng, J.T., 2014. ER stress, autophagy, and RNA viruses. Frontiers in microbiology 5,388 .

Kumar, S., Gupta, P., Khanal, S., Shahi, A., Kumar, P., Sarin, S.K., Venugopal, S.K., 2015. Over-expression of microRNA-30a Inhibits Hepatitis B Virus X Protein-Induced Autophagosome Formation in Hepatic Cells. The FEBS journal.

Lee, Y.R., Lei, H.Y., Liu, M.T., Wang, J.R., Chen, S.H., Jiang-Shieh, Y.F., Lin, Y.S., Yeh, T.M., Liu, C.C., Liu, H.S., 2008. Autophagic machinery activated by dengue virus enhances virus replication. Virology 374, 240-248.

Lee, Y.R., Wang, P.S., Wang, J.R., Liu, H.S., 2014. Enterovirus 71-induced autophagy increases viral replication and pathogenesis in a suckling mouse model. Journal of biomedical science 21 , 
80.

Lussignol, M., Queval, C., Bernet-Camard, M.F., Cotte-Laffitte, J., Beau, I., Codogno, P., Esclatine, A., 2013. The herpes simplex virus 1 Us11 protein inhibits autophagy through its interaction with the protein kinase PKR. Journal of virology 87, 859-871.

McMinn, P.C., 2002. An overview of the evolution of enterovirus 71 and its clinical and public health significance. FEMS microbiology reviews 26, 91-107.

Mizushima, N., Yamamoto, A., Matsui, M., Yoshimori, T., Ohsumi, Y., 2004. In vivo analysis of autophagy in response to nutrient starvation using transgenic mice expressing a fluorescent autophagosome marker. Molecular biology of the cell 15, 1101-1111.

Mizushima, N., Yoshimori, T., Levine, B., 2010. Methods in mammalian autophagy research. Cell $140,313-326$.

Munz, C., 2011. Beclin-1 targeting for viral immune escape. Viruses 3, 1166-1178.

Nikoletopoulou, V., Papandreou, M.E., Tavernarakis, N., 2015. Autophagy in the physiology and pathology of the central nervous system. Cell death and differentiation 22, 398-407.

Nixon, R.A., Cataldo, A.M., Mathews, P.M., 2000. The endosomal-lysosomal system of neurons in Alzheimer's disease pathogenesis: a review. Neurochemical research 25, 1161-1172.

Pan, W., Zhong, Y., Cheng, C., Liu, B., Wang, L., Li, A., Xiong, L., Liu, S., 2013. MiR-30-regulated autophagy mediates angiotensin II-induced myocardial hypertrophy. PloS one 8, e53950.

Pattingre, S., Espert, L., Biard-Piechaczyk, M., Codogno, P., 2008. Regulation of macroautophagy by mTOR and Beclin 1 complexes. Biochimie 90, 313-323.

Poojary, V.K., Xi, X., Zhang, X., Wang, B., Wang, T., Wang, J., Huang, H., Wang, J., Jin, Q., Zhao, Z., 2013. The Interplays between Autophagy and Apoptosis Induced by Enterovirus 71. PloS one 8, e56966.

Richards, A.L., Jackson, W.T., 2013. How positive-strand RNA viruses benefit from autophagosome maturation. Journal of virology 87, 9966-9972.

Schlegel, A., Giddings, T.H., Jr., Ladinsky, M.S., Kirkegaard, K., 1996. Cellular origin and ultrastructure of membranes induced during poliovirus infection. Journal of virology 70, 6576-6588.

Skalsky, R.L., Cullen, B.R., 2010. Viruses, microRNAs, and host interactions. Annual review of 
microbiology 64, 123-141.

Solomon, T., Lewthwaite, P., Perera, D., Cardosa, M.J., McMinn, P., Ooi, M.H., 2010. Virology, epidemiology, pathogenesis, and control of enterovirus 71. The Lancet. Infectious diseases 10, 778-790.

Suhy, D.A., Giddings, T.H., Jr., Kirkegaard, K., 2000. Remodeling the endoplasmic reticulum by poliovirus infection and by individual viral proteins: an autophagy-like origin for virus-induced vesicles. Journal of virology 74, 8953-8965.

Taylor, M.P., Jackson, W.T., 2009. Viruses and arrested autophagosome development. Autophagy $5,870-871$.

Wang, P., Liang, J., Li, Y., Li, J., Yang, X., Zhang, X., Han, S., Li, S., Li, J., 2014. Down-regulation of miRNA-30a alleviates cerebral ischemic injury through enhancing beclin 1-mediated autophagy. Neurochemical research 39, 1279-1291.

Wong, J., Zhang, J., Si, X., Gao, G., Mao, I., McManus, B.M., Luo, H., 2008. Autophagosome supports coxsackievirus B3 replication in host cells. Journal of virology 82, 9143-9153.

Wong, S.S., Yip, C.C., Lau, S.K., Yuen, K.Y., 2010. Human enterovirus 71 and hand, foot and mouth disease. Epidemiology and infection 138, 1071-1089.

Xu, L.J., Jiang, T., Zhao, W., Han, J.F., Liu, J., Deng, Y.Q., Zhu, S.Y., Li, Y.X., Nian, Q.G., Zhang, Y., Wu, X.Y., Qin, E.D., Qin, C.F., 2014. Parallel mRNA and microRNA profiling of HEV71-infected human neuroblastoma cells reveal the up-regulation of miR-1246 in association with DLG3 repression. PloS one 9, e95272.

Yamamoto, A., Yue, Z., 2014. Autophagy and its normal and pathogenic states in the brain. Annual review of neuroscience $37,55-78$.

Yin, X., Peng, C., Ning, W., Li, C., Ren, Z., Zhang, J., Gao, H., Zhao, K., 2013. miR-30a downregulation aggravates pressure overload-induced cardiomyocyte hypertrophy. Molecular and cellular biochemistry $379,1-6$.

Yoshimori, N.M.T., 2007. How to Interpret LC3 Immunoblotting. Autophagy 3:6, 542-545.

Yu, Y., Yang, L., Zhao, M., Zhu, S., Kang, R., Vernon, P., Tang, D., Cao, L., 2012. Targeting microRNA-30a-mediated autophagy enhances imatinib activity against human chronic myeloid leukemia cells. Leukemia 26, 1752-1760.

Zalckvar, E., Yosef, N., Reef, S., Ber, Y., Rubinstein, A.D., Mor, I., Sharan, R., Ruppin, E., Kimchi, 
A., 2010. A systems level strategy for analyzing the cell death network: implication in exploring the apoptosis/autophagy connection. Cell death and differentiation 17, 1244-1253.

Zhai, H., Fesler, A., Ju, J., 2013. MicroRNA: a third dimension in autophagy. Cell cycle 12, 246-250.

Zhang, H., Li, F., Pan, Z., Wu, Z., Wang, Y., Cui, Y., 2014a. Activation of PI3K/Akt pathway limits JNK-mediated apoptosis during EV71 infection. Virus research 192, 74-84.

Zhang, L., Chen, X., Shi, Y., Zhou, B., Du, C., Liu, Y., Han, S., Yin, J., Peng, B., He, X., Liu, W., 2014b. miR-27a suppresses EV71 replication by directly targeting EGFR. Virus genes.

Zheng, Z., Ke, X., Wang, M., He, S., Li, Q., Zheng, C., Zhang, Z., Liu, Y., Wang, H., 2013. Human microRNA hsa-miR-296-5p suppresses enterovirus 71 replication by targeting the viral genome. Journal of virology 87, 5645-5656.

Zhou, Z., Jiang, X., Liu, D., Fan, Z., Hu, X., Yan, J., Wang, M., Gao, G.F., 2009. Autophagy is involved in influenza A virus replication. Autophagy 5, 321-328.

Zou, Z., Wu, L., Ding, H., Wang, Y., Zhang, Y., Chen, X., Chen, X., Zhang, C.Y., Zhang, Q., Zen, K., 2012. MicroRNA-30a sensitizes tumor cells to cis-platinum via suppressing beclin 1-mediated autophagy. The Journal of biological chemistry 287, 4148-4156.

\section{Figure legends:}

Figure-1: EV71-induced autophagy activity in Hep2 and Vero cells.

(A) Formation of GFP-LC3 puncta in EV71-infected Hep2 (upper panel) and Vero cells (lower panel) with or without the presence of wortmannin (300 nM for hep2 and $100 \mathrm{nM}$ for Vero cells). Cells were transfected with EGFP-LC3 plasmid for $12 \mathrm{~h}$, followed by EV71 infection at 0.03 $\mathrm{TCID}_{50}$ for indicated times. The percentage of the cells with punctated GFP-LC3 was expressed as cells with three or more puncta vs total GFP positive cells. Red arrows indicate the GFP-LC3 puncta. (B) EV71-induced autophagy by transmission electron microscopy. White arrows indicate autophagosome-like structures. $\mathrm{N}$ : nucleus. Right panel shows quantification of the average number of autophagosome-like vesicles per Vero cell and a minimum of 50 cells were counted. (C) Colocalization of LC3-II and VP1 in Hep2 cells transfected with GFP-LC3 plasmid and infected 
with EV71 at a $0.03 \mathrm{TCID}_{50} .24$ hours pi, cells were stained with anti-EV71 mAb and Alexa Fluor 546-conjugated anti-mouse $\operatorname{IgG}$ antibody. Images were acquired using a confocal microscope. $(* \mathrm{P}<0.05, * * \mathrm{P}<0.01, * * * \mathrm{P}<0.001)$.

Figure-2: Protein expressions relevant to autophagic activity were altered upon EV71 infection and autophagy facilitated viral replication

(A, B) Western analysis of the expression levels of Beclin-1, VP1, LC3-I and LC3-II in Hep2 (A) and Vero cells (B) infected with EV71 for indicated time in the presence or absence of Wortmannin. The numbers represent the relative density of the band in comparison to the corresponding control normalized to $\beta$-acting. Value of mock treatment is set at $1.00(100 \%)$. (C, D) Titers of progeny EV71 in the culture supernatant of infected-cells in the presence or absence of wortmannin. $(* \mathrm{P}<0.05)$.

Figure-3: EV71 infection down-regulated expression of hsa-miR-30a.

The expression of hsa-miR-30a and miR-18a in infected cells with or without the presence of wortmannin is quantified by qRT-PCR, using U6 rRNA as an internal control. The final concentrations of wortmannin are $100 \mathrm{nM}$ and $300 \mathrm{nM}$ in Vero and Hep2 cells, respectively. Values are mean of triplicate experiments and presented in relative to the level of Mock infection without wortmannin in Hep2 (A) and Vero (B) cells. Mock infection without wortmannin is assigned with a value of 1 . The data are presented as mean \pm SEM $(\mathrm{n}=3, * \mathrm{P}<0.05, * * \mathrm{P}<0.01$, $* * * \mathrm{P}<0.001)$.

\section{Figure-4: miR-30a targets and inhibits Beclin-1}

The putative miR-30a binding sites of nt $98-105$ in Beclin-13'-UTR region as predicted by TargetScan (red) and the mutation introduced by site-directed mutagenesis are shown (underline) (A). The luciferase activity of Beclin-1 3'UTR is repressed by transfected miR-30a in Hep2 (B) and Vero cells (C). Mutation of a predicted miR-30a binding site in the Beclin-1 3'UTR abolished miR-30a-mediated repression (B, C). After transfection with pre-miR-30a, antago-miR-30a or their respective controls for 48 hours, the expression levels of miR-30a in Hep2 and Vero cells (D) are determined by qRT-PCR and normalized to U6 rRNA level. The expression of Beclin-1, p62 
and LC3 is measured by Western blot analysis in Hep2 (E) and Vero cells (F) transfected with respective miRNA plasmids. The numbers denote the relative density of the bands normalized to the control. The Beclin-1 mRNA is determined by qRT-PCR and normalized to $\beta$-actin mRNA levels in Hep2 and Vero cells $(\mathrm{G})$. The data are presented as mean \pm SEM $(\mathrm{n}=3, *, \# \mathrm{P}<0.05$, $* *, \# \# \mathrm{P}<0.01, * * *, \# \# \mathrm{P}<0.001)$.

\section{Figure-5: Overexpression of hsa-miR-30a inhibited EV71 replication}

Extracellular and intracellular infectious virus production of EV71 in cells transfected with pre-miR-30a or negative control (n.c). Data are mean \pm SEM of fold change and representative of at least three independent experiments (A,B). Hep2 cells are transfected with pre-miR-30a or negative control (n.c) for $24 \mathrm{~h}$ and then infected with EV71. Infected cells are identified by staining with anti-VP1 antibody and Alexa Fluor 546-conjugated IgG antibody. Nuclei are identified by staining with DAPI. Data points presented are average from six different fields (C). The expression levels of VP1, Beclin-1, LC3-I and LC3-II in pre-miR-30a-transfected cells infected with EV71 (D). The protein bands are visualized by specific antibodies and the numbers denote the relative density of the bands normalized to the Mock cells transfected with pre-miR-n.c which is assigned a value of 1. GFP-LC3 puncta in pre-miR-30a-transfected cells with or without EV71 infection (E). $(* \mathrm{P}<0.05, * * \mathrm{P}<0.01)$.

\section{Figure-6: Inhibition of hsa-miR-30a promotes EV71 replication.}

Titers of progeny EV71 in cells transfected with antago-miR-30a or negative control (n.c) are determined as described in the text. Each datum is mean \pm SEM of treplicate experiment (A). Viral protein VP1 in antago-miR-30a-transfected cells during EV71 infection is visualized using specific antibodies and scanned with a densitometer(B). Cells are transfected with antago-miR-30a or negative control (n.c) for $24 \mathrm{~h}$ and then infected with EV71. Infected cells are identified by staining with anti-VP1 antibody and nuclei were identified by staining with DAPI. The quantification represents the average from six different fields $(\mathrm{C}) .\left({ }^{*} \mathrm{P}<0.05\right)$. 
Figure(s)

Figure-1
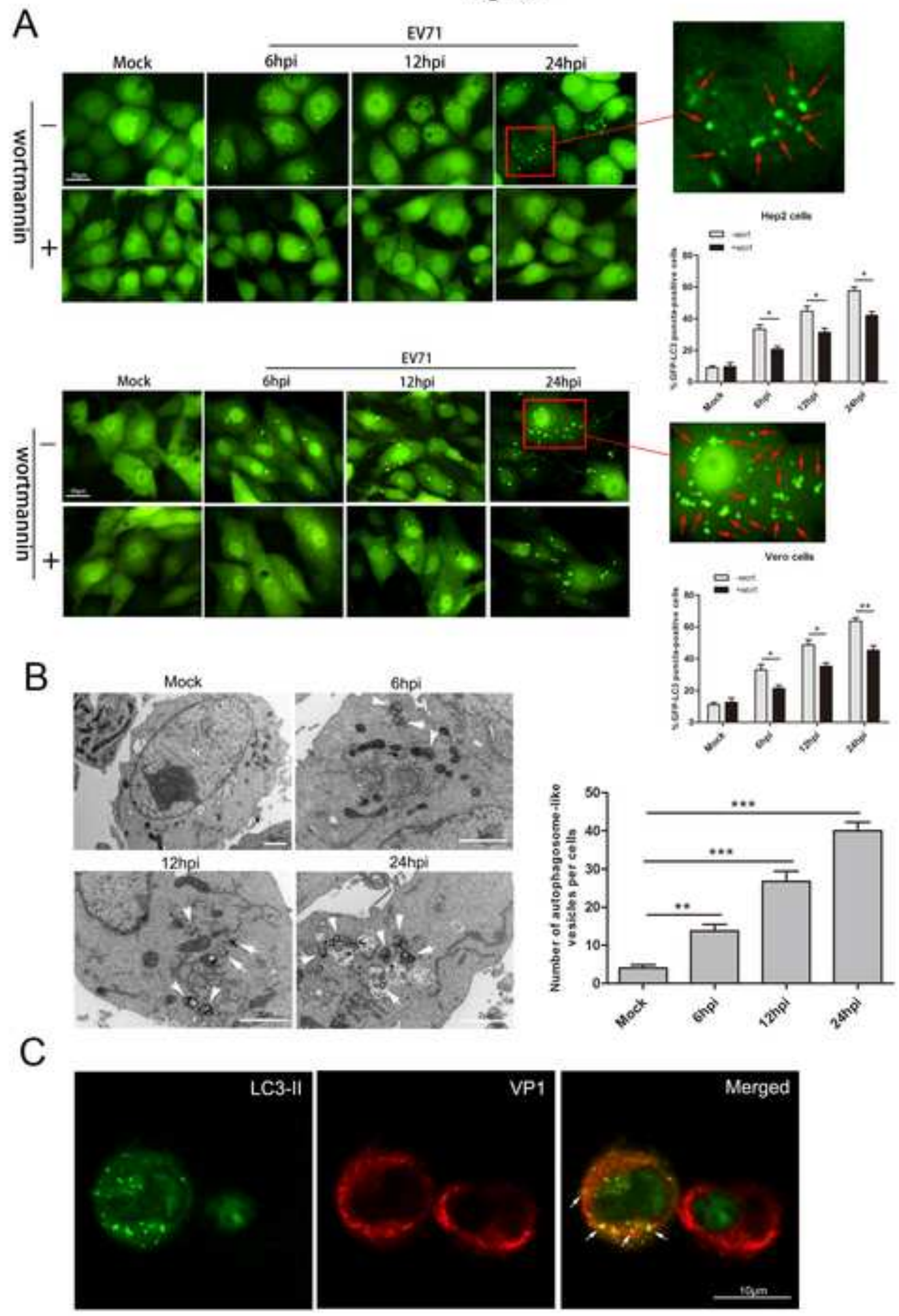

C
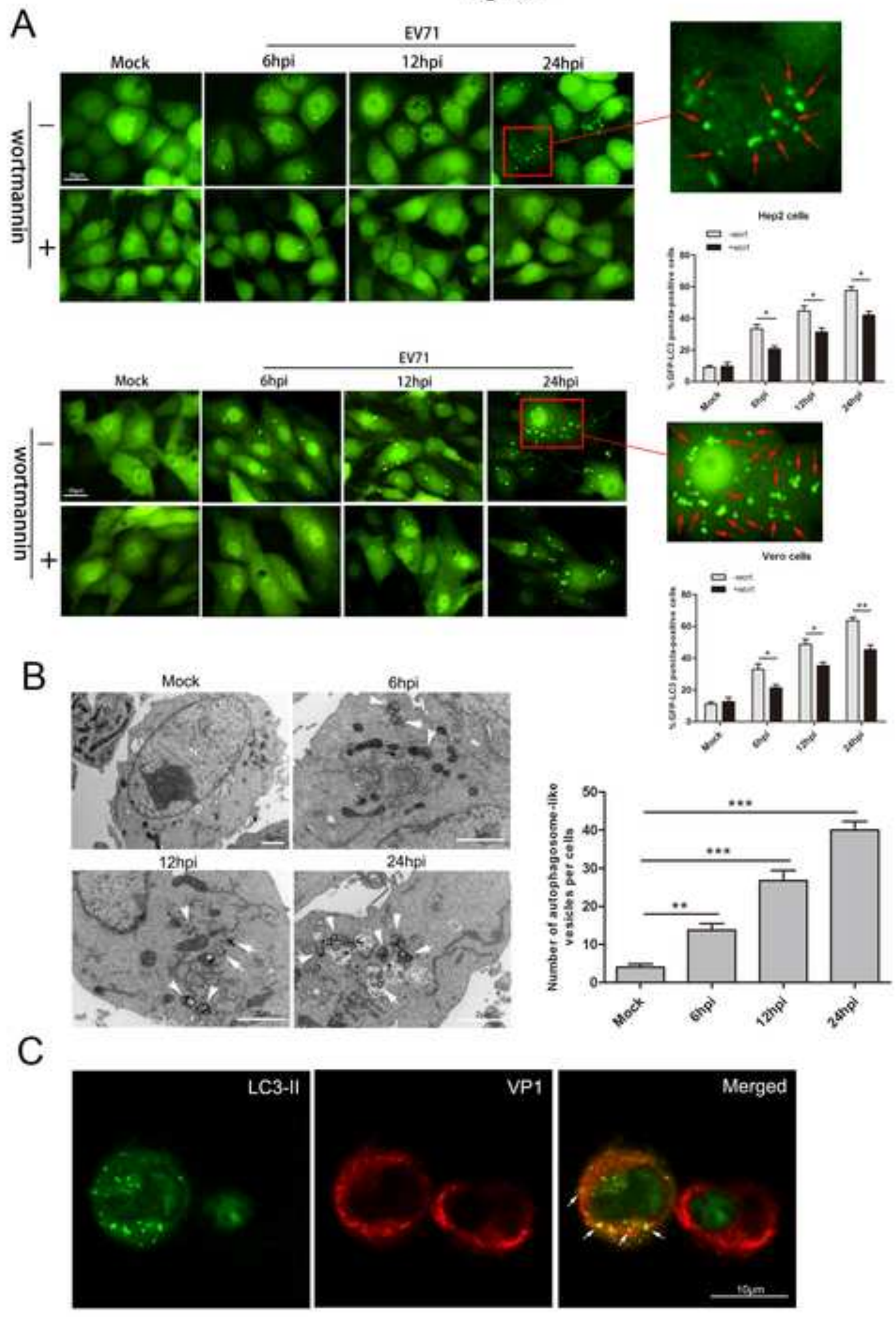
Figure-2

A

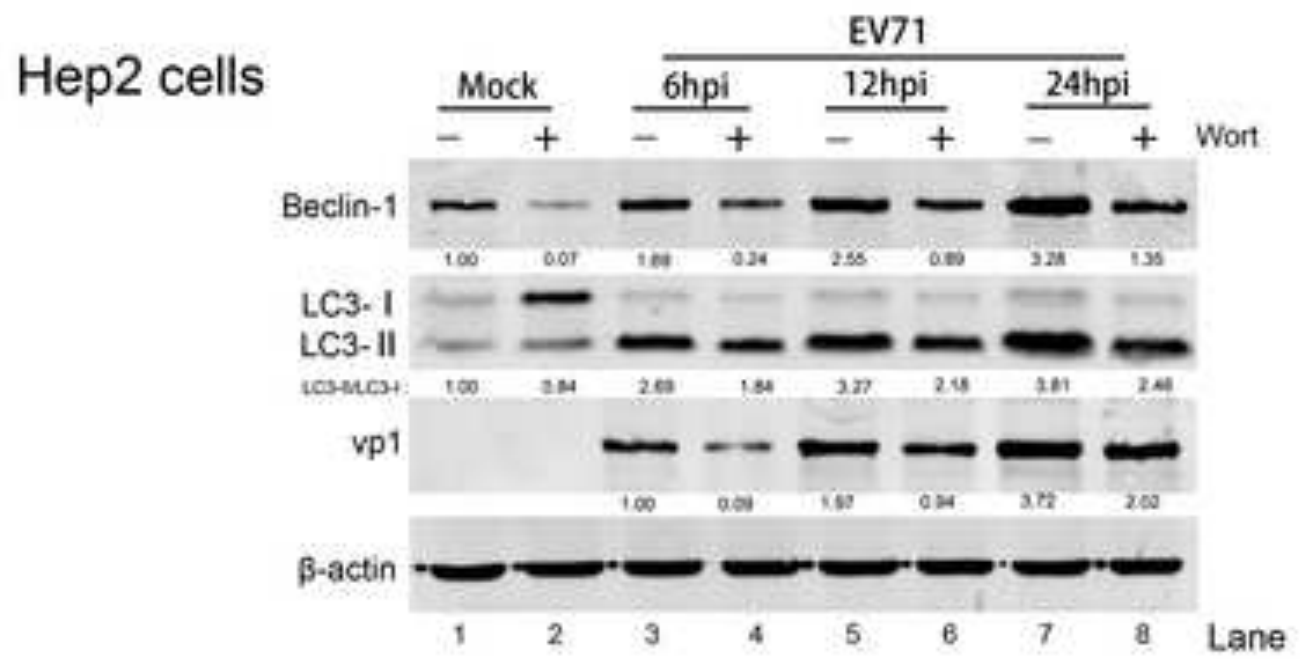

B

Vero cells
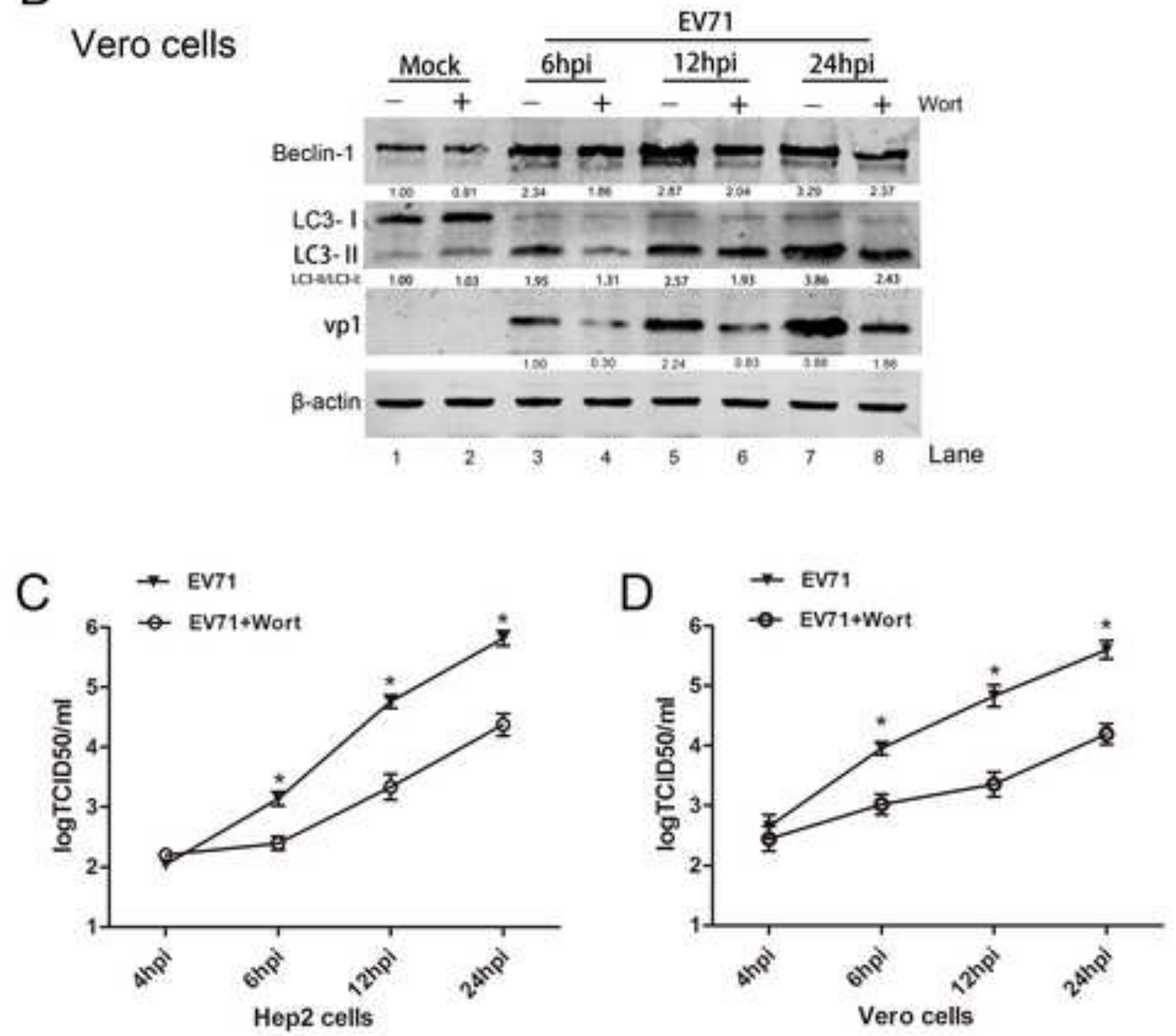
A Hep2 cells

Figure-3

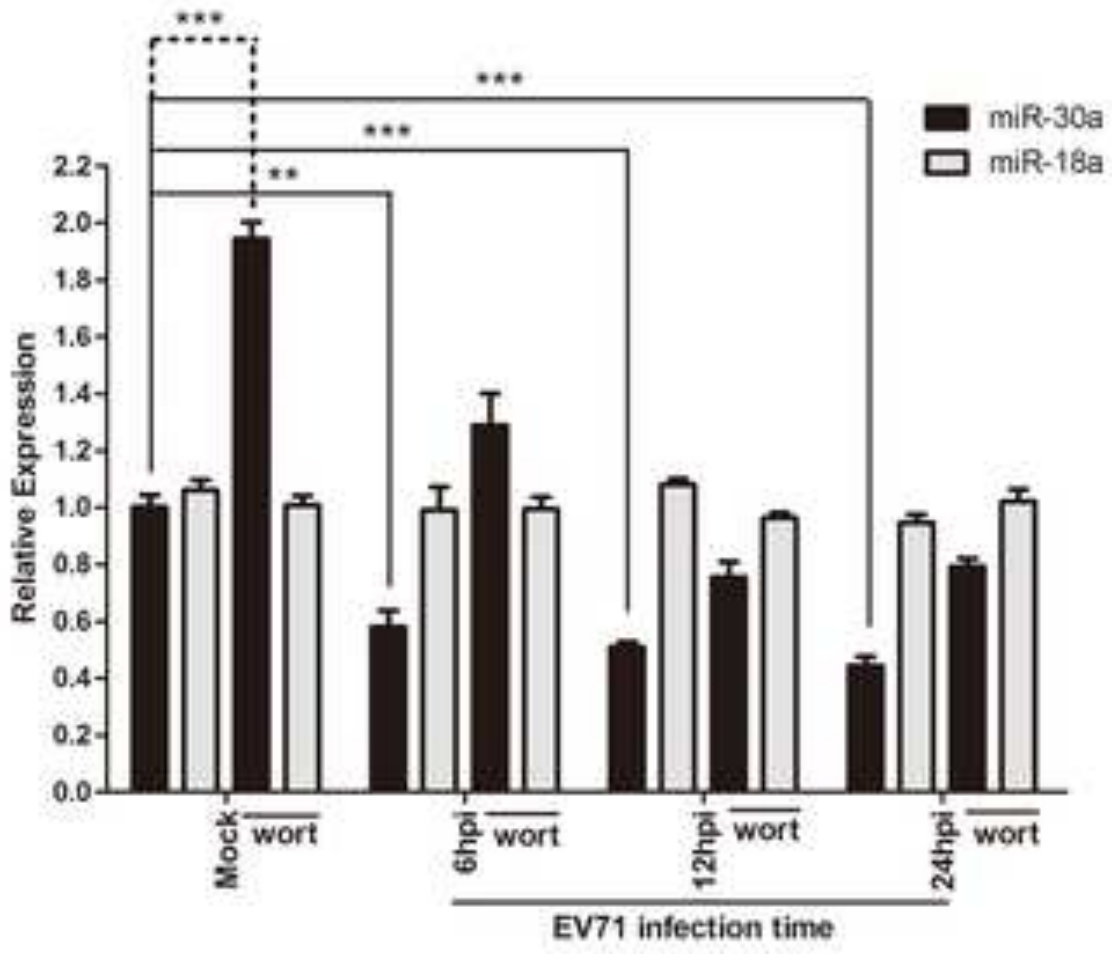

B

Vero cells

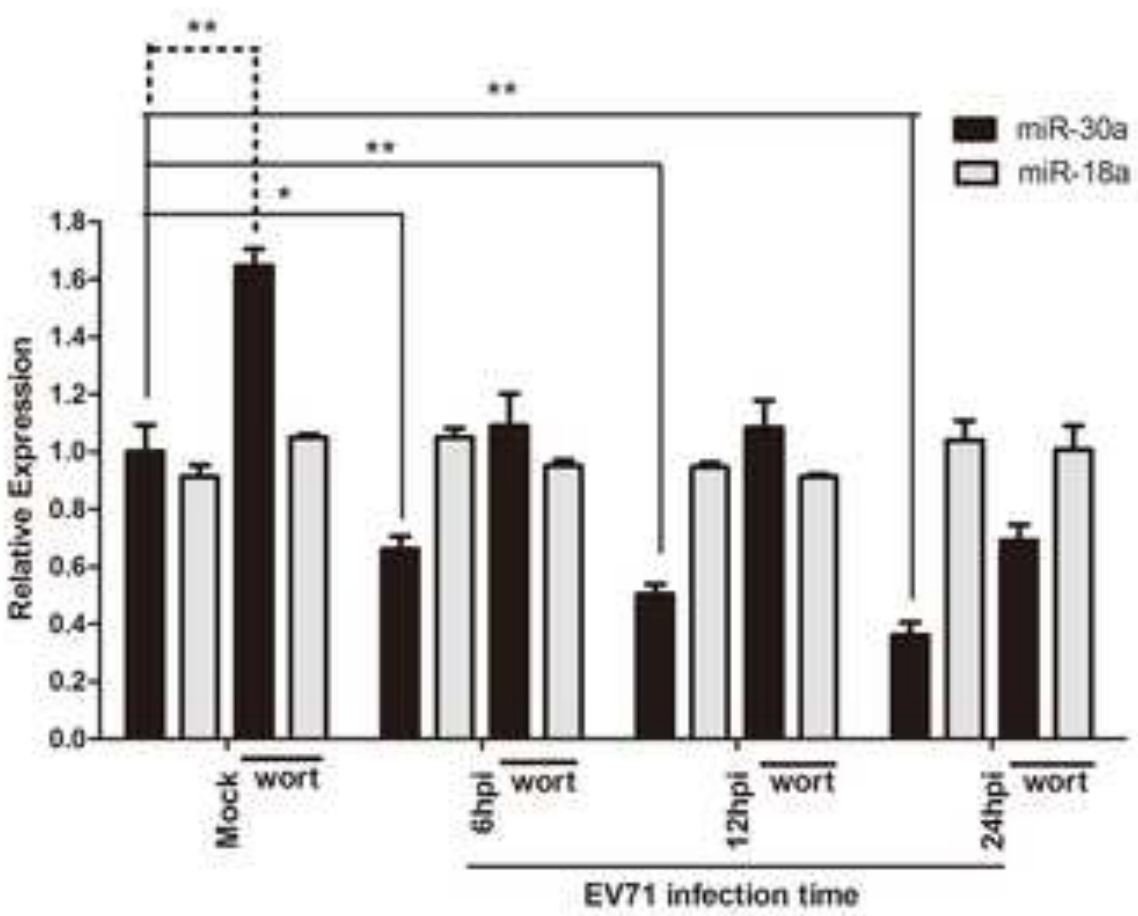


Figure(s)

Figure-4

A

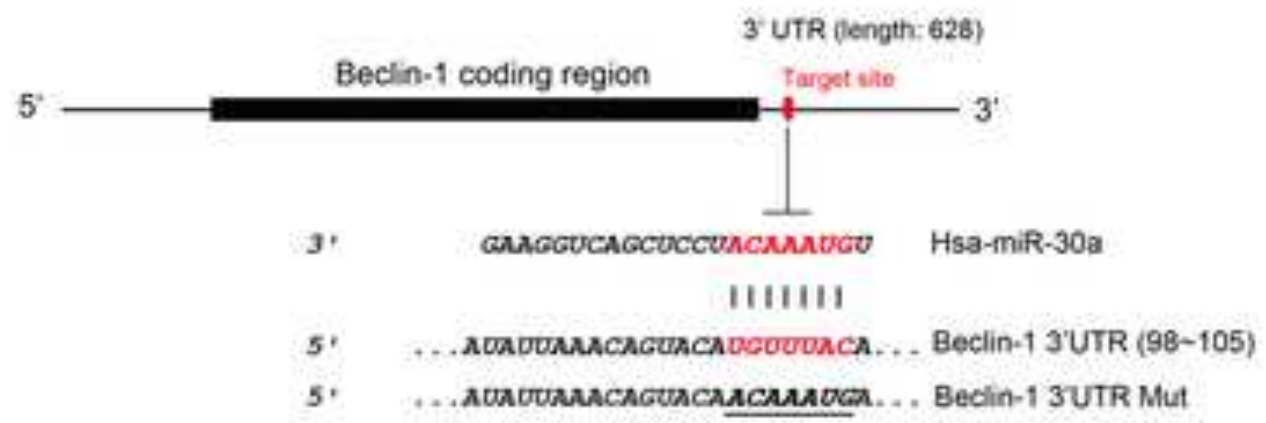

B

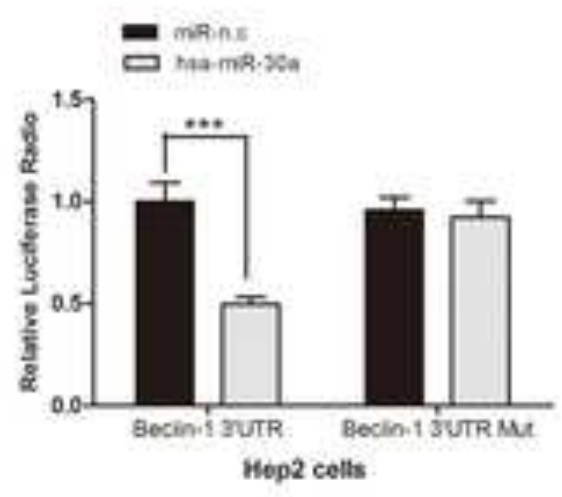

D

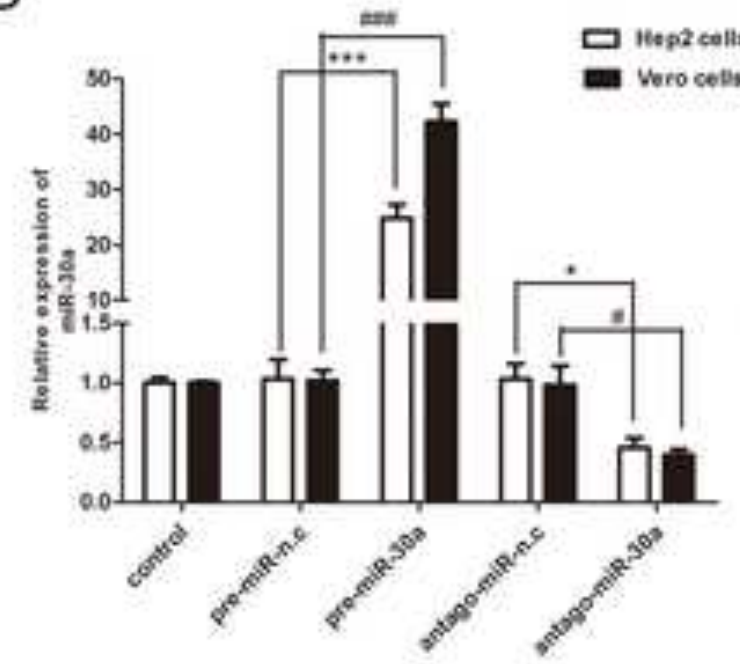

G 므 Noro cells

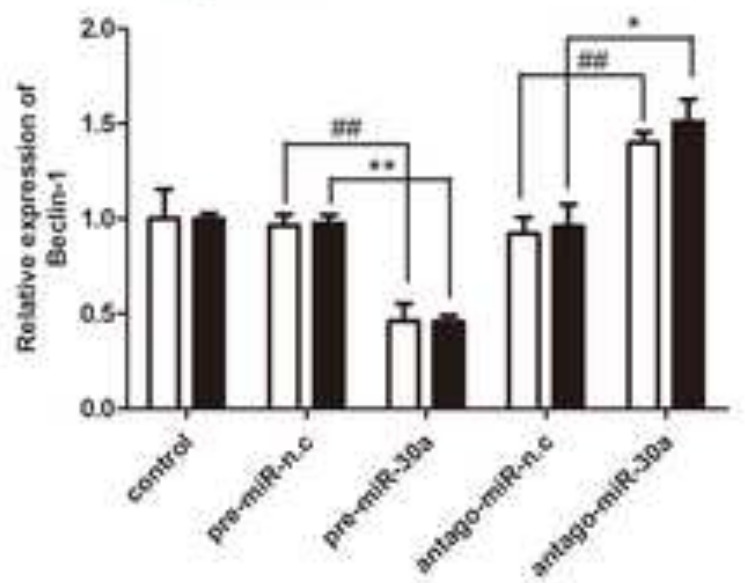

C

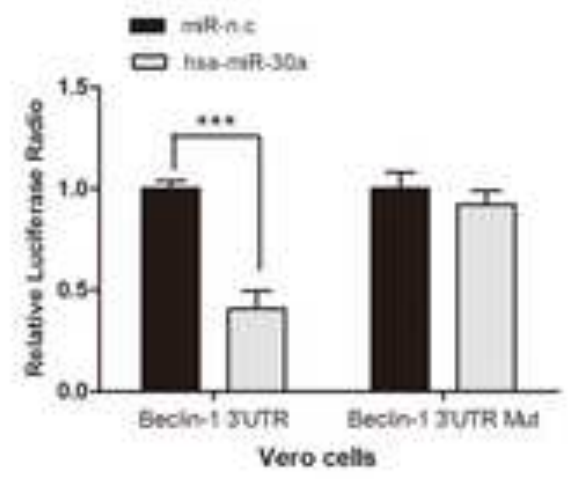

E

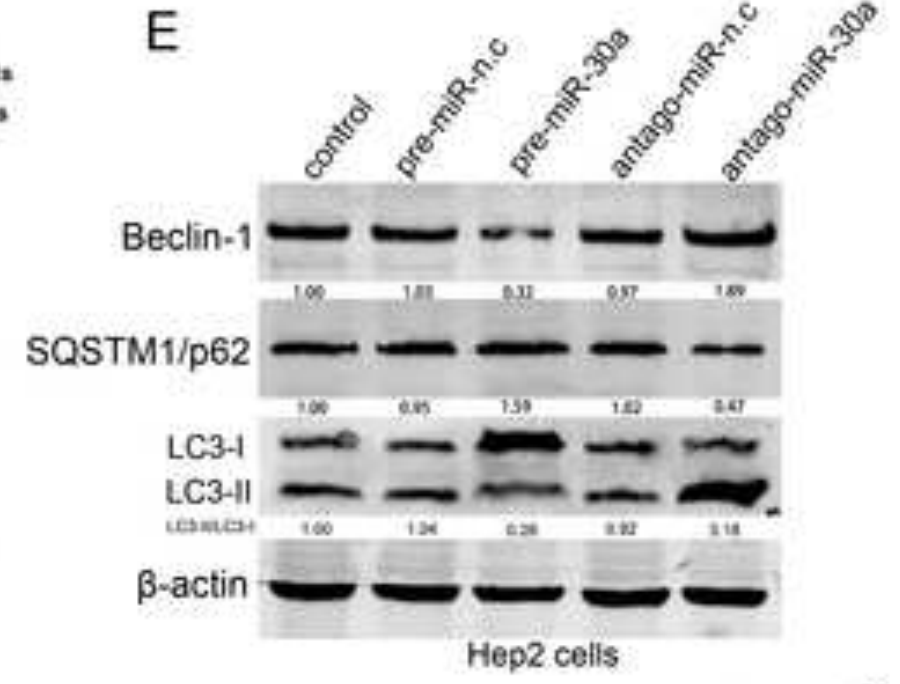

F

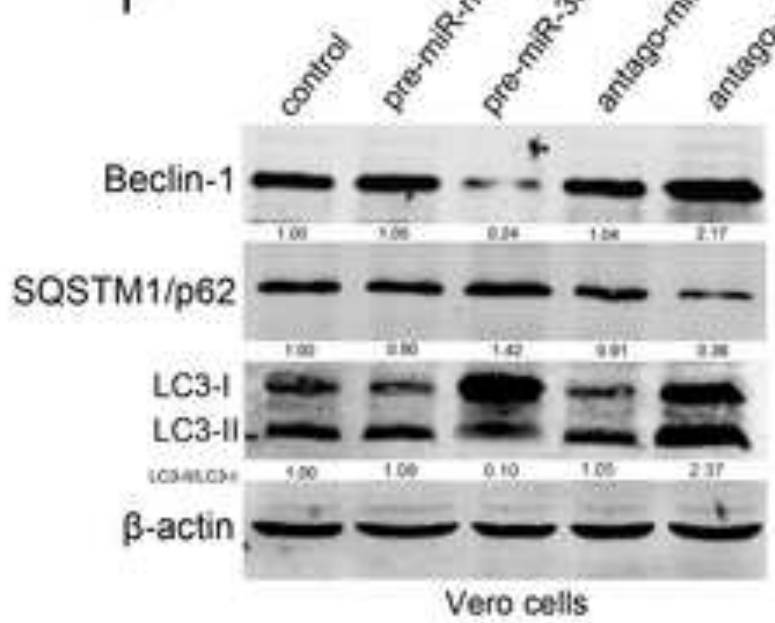


A

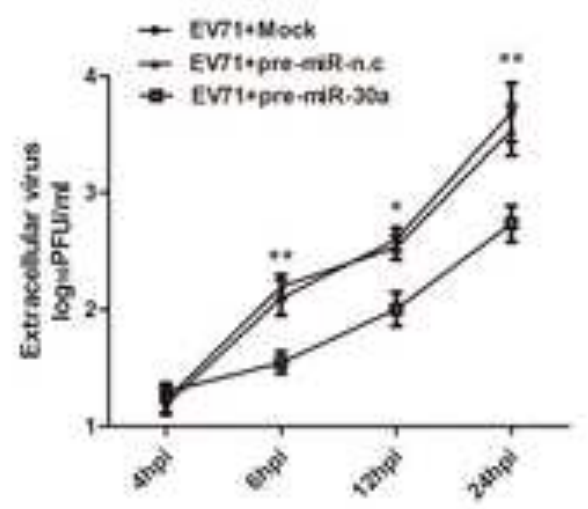

C

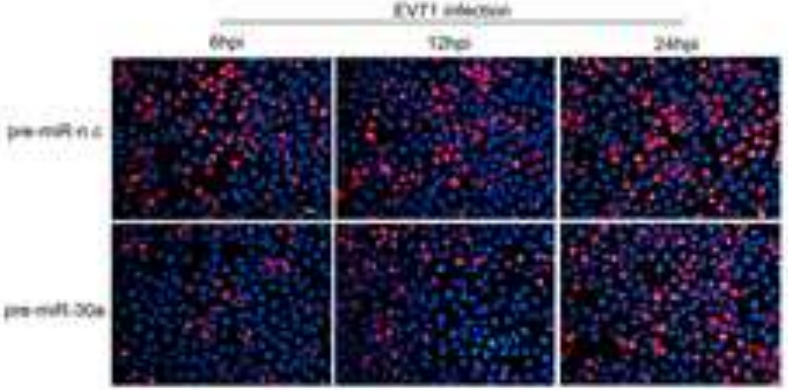

D

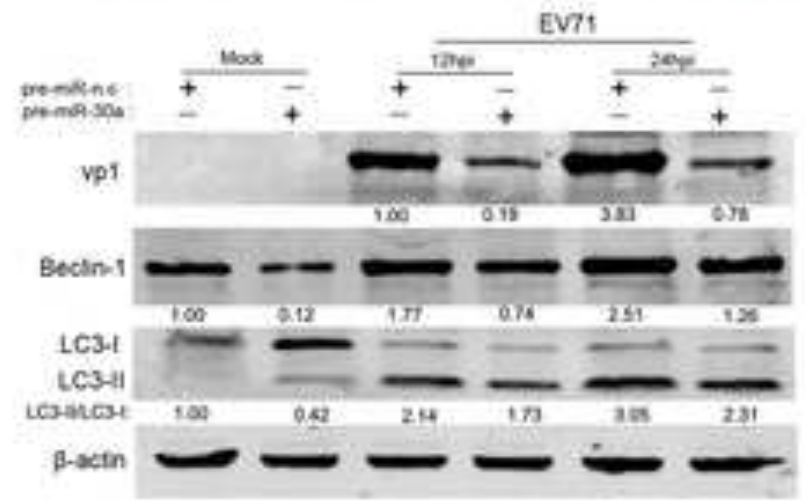

E

EV71
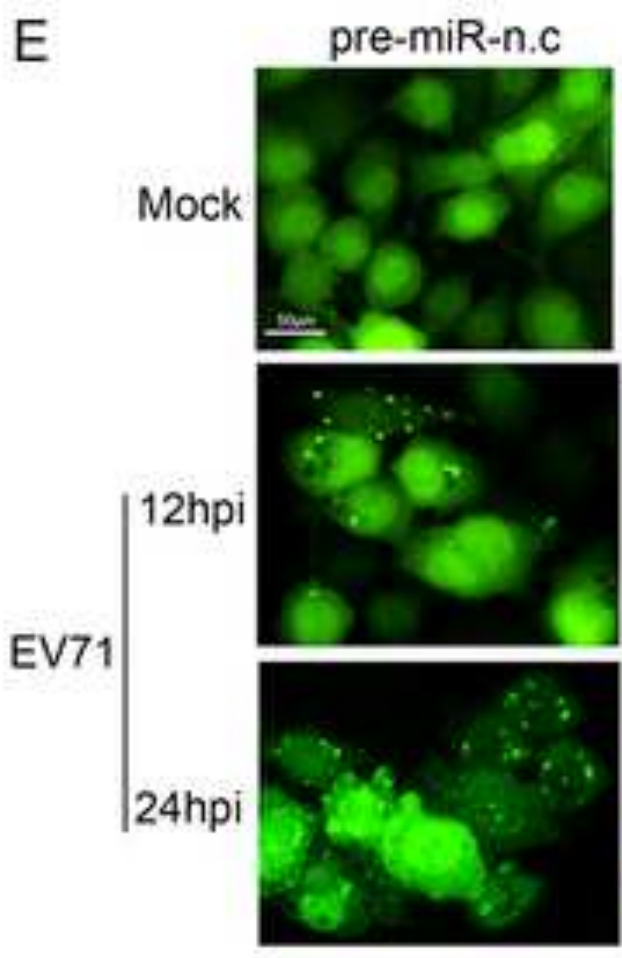
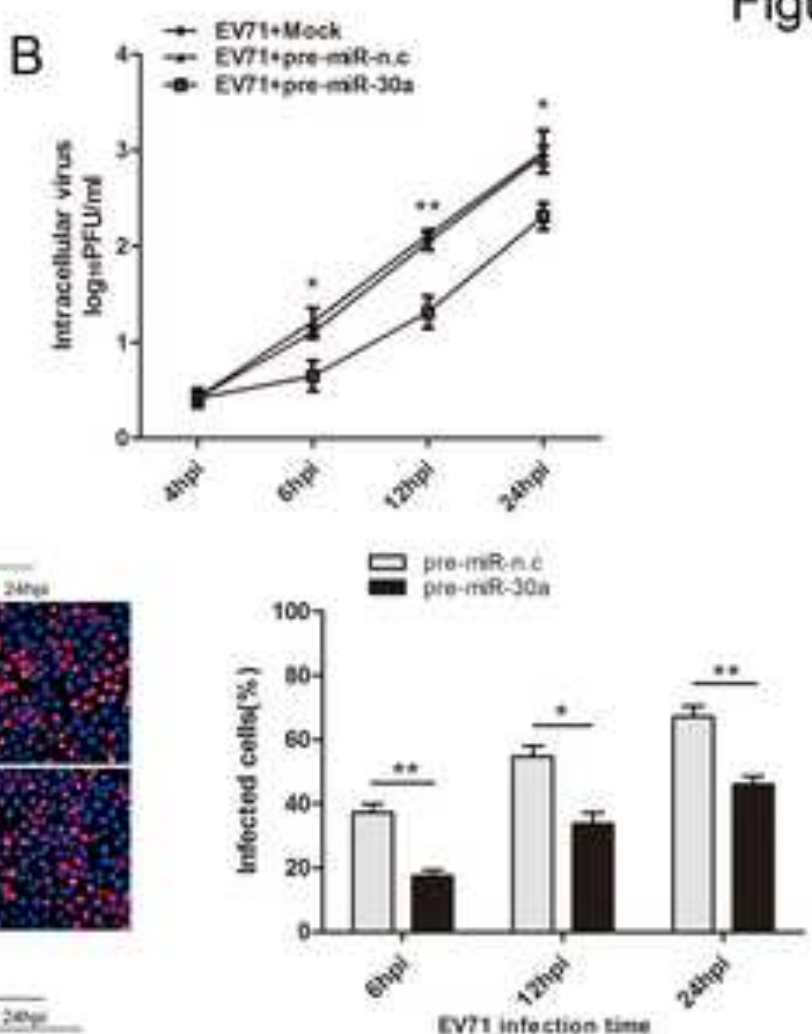

Figure-5
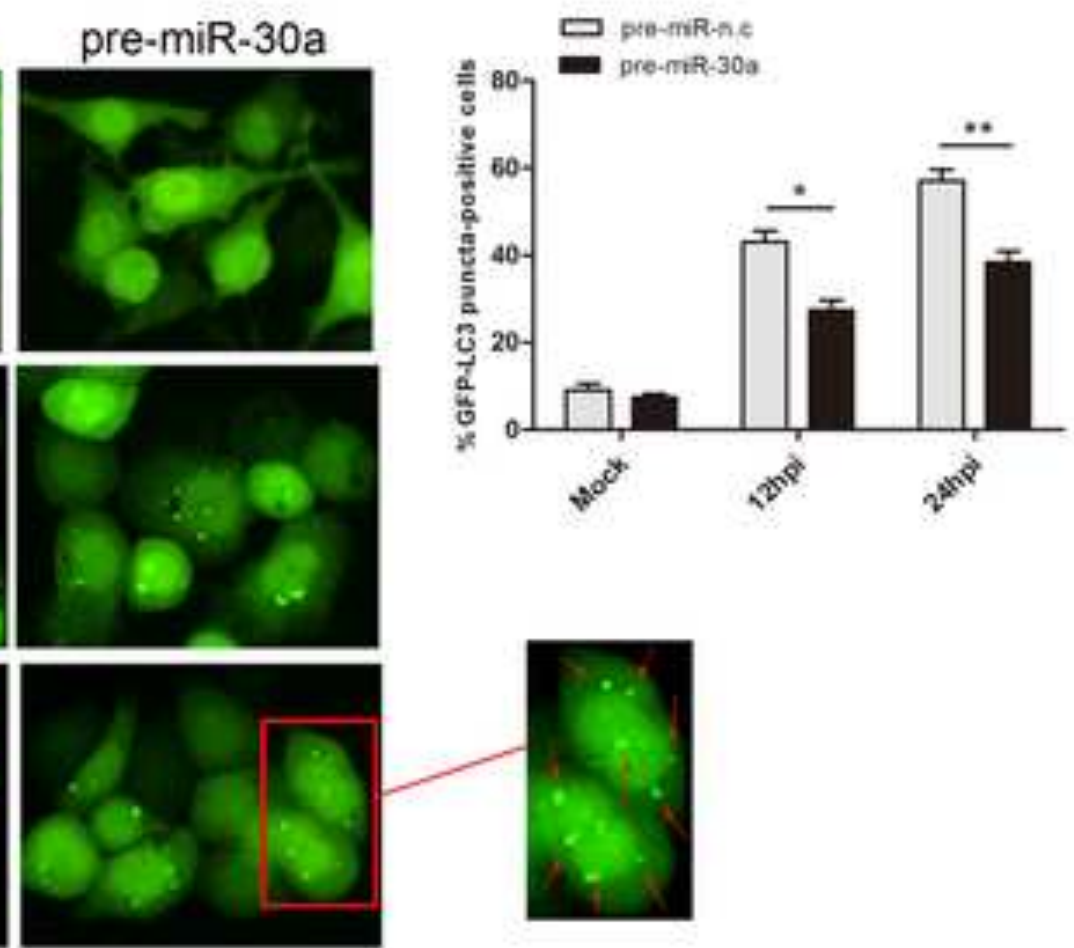
A

Figure-6

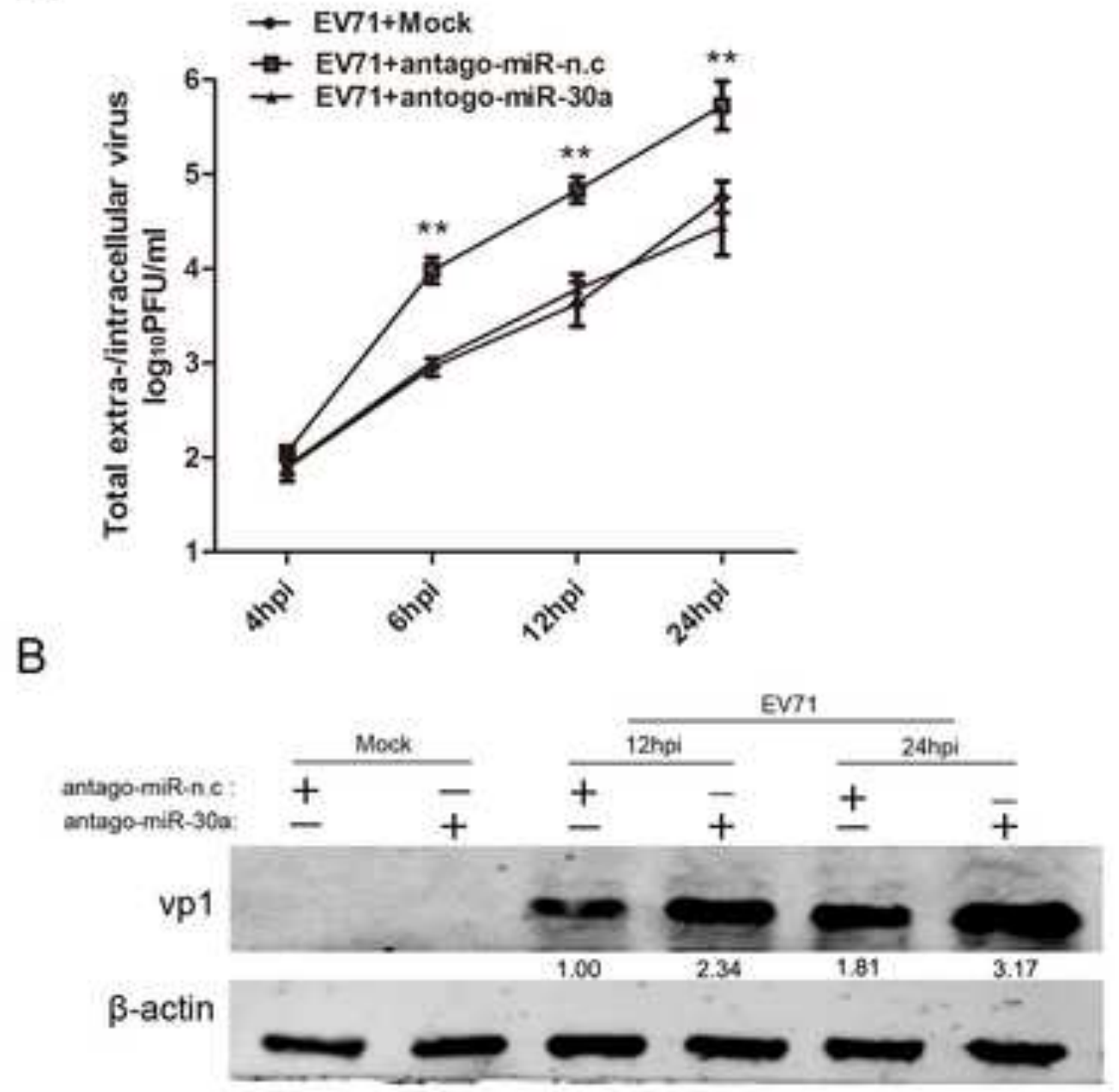

C
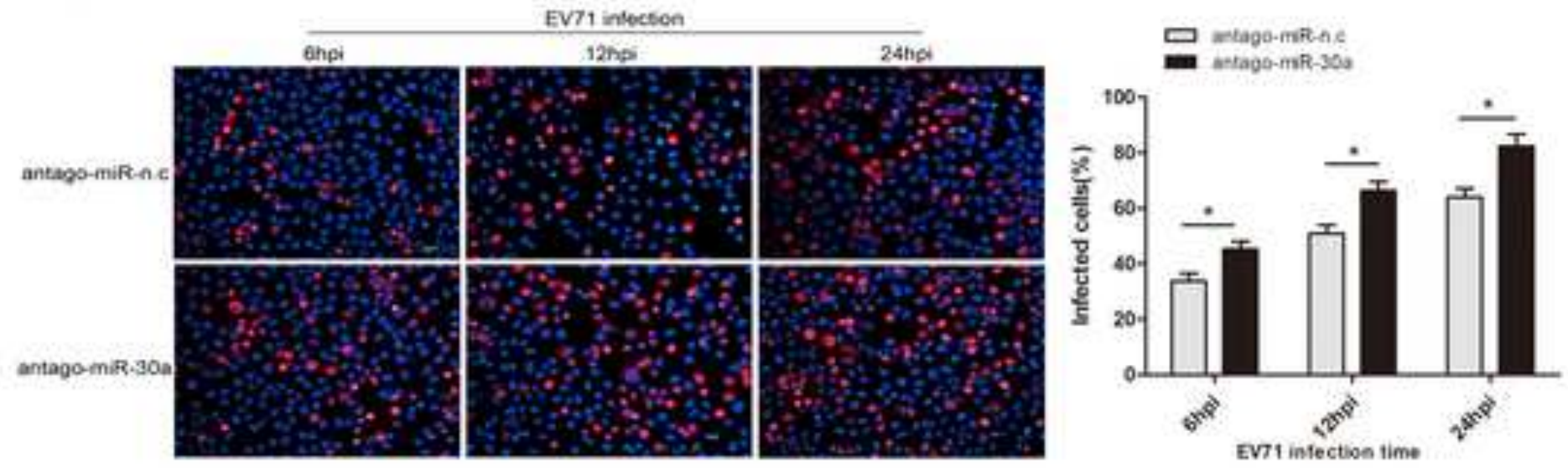